\title{
Analysis of seismic tomography data for definition of hydraulic fracturing parameters in underground coal mines
}

\author{
Gennadii Rout ${ }^{1 *}$, Sergey Sokolov ${ }^{2}$, Evgeniy Utkaev $^{2}$, and Kolmakova Anastasiya ${ }^{2}$ \\ ${ }^{1}$ T.F. Gorbachev Kuzbass State Technical University \\ ${ }^{2}$ Federal State Budget Scientific Centre «The Federal Research Center of Coal and Coal-Chemistry of \\ Siberian Branch of the Russian Academy of Sciences)
}

\begin{abstract}
The article analyzes the issue of reliable estimate of the unloading extent and the variation order of coal-rock massif geomechanical characteristics as a result of hydraulic fracturing undertaken from mine workings. For this purpose it is proposed to use the fracturing value of the studied rock mass. In case the possibility of comparison with geological and actual data doesn't exist, the option of using the rock mass classification based on the estimated value of geophysical index that specifies rock mass fracturing is considered. To address the issue, a geophysical survey of the active roof at the excavation site in the operating coal mine was implemented with a method of a seismic radioscopy before and after hydraulic fracturing. According to the results of seismic exploration, a massif unloading degree and an extent of roof fracturing has been determined.
\end{abstract}

\section{Introduction}

The use of hydraulic fracturing in coal mines can meet different requirements. Hydraulic action is distinguished in order to ensure the intensification of methane recovery from coal seams, and to eliminate the negative effect of a difficult-to-collapse roof [1-4]. For each of the listed objectives fracturing processes are characteristic and, as a consequence, changes in the geomechanical composition of rocks in the area of hydraulic impact are observed. To assess the indicated area propagation, the method of seismic tomography on transmitted waves, based on the interpretation of velocity characteristics of the seismotomographic cross section $[5,6]$, is becoming widely used among other tools to control hydraulic fracturing parameters. The use of seismic tomography makes it possible to establish the presence of contrasting regions characterized by a change in the physical and mechanical state of the massif [7]. At the same time, in the course of measurement results presentation to the consumer, the most difficult point lies in reliably of results on the assed degree of massif unloading and in the order of geomechanical characteristics changes.

\footnotetext{
${ }^{*}$ Corresponding author: routgn@,kuzstu.ru
} 


\section{Methodologies}

When carrying out geophysical study, a set of seismic equipment (Figure 1) was used to record data, consisting of: autonomous seismic recorders "R-1", providing seismic data in digital format (1) and geophones (2), determining the number of channels in the performed research. A percussive source of mechanical vibrations is used to generate the signal.

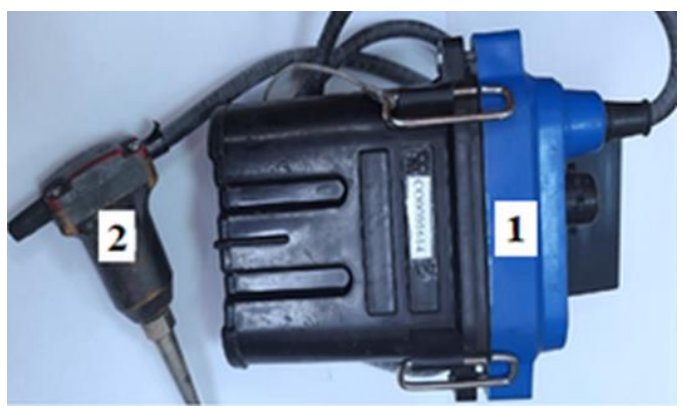

Fig. 1. A set of recording equipment to implement measurements with a seismic tomography method.

To assess the instantaneous velocities in different sections of studied extraction column, analytical approaches were used for tomographic examination of a massif section contoured by two workings and divided in accordance with the parameters of the applied measurement scheme into $\mathrm{N}$ pixels using the relationship [8]

$$
t_{i}=\sum_{j=1}^{N} \frac{1}{V_{j}} d_{i j} \quad(\mathrm{i}=1 \ldots \mathrm{n})
$$

where $\mathrm{t}$ and $\mathrm{V}$ are the time and propagation velocity of a seismic wave; 1 is the distance from the oscillation source to the receiver; $\mathrm{n}$ is the number of observations; $d_{i j}$ - travel distance of the ray $i$ in pixel $j$.

For the numerical assessment of roof softening under hydraulic fracturing application, the fracturing of the investigated rock mass was chosen as a criterion, which means the ratio of cracks amount and voids in the investigated massif to the bulk of rock skeleton. In this case, the calculation accepted the dependence of the longitudinal wave velocity on the porosity coefficient, which is the ratio of the pore volume to the rock skeleton bulk [9]:

$$
\frac{1}{V_{p}}=\frac{k}{V_{\phi}}+\frac{1-k}{V_{\Pi}}
$$

where $k$ is the coefficient of porosity, regarding the presence of cracks, pores and voids of the rock under study; $V_{p}$ is the velocity of the longitudinal wave in the investigated massif; $V_{\phi}$ is the velocity of the longitudinal wave in the fluid; $V_{\Pi}$ - the velocity of the longitudinal wave in the rock skeleton.

In this particular case of longitudinal waves reception and excitation, the influence on signal parameters of the entire volume of differently oriented cracks formed as a result of softening, resulted due to the difference in elevation marks of parallel workings and the alternation of vibration excitation points was accepted. To assess changes in roof conditions caused by hydraulic fracturing, instead of $k$ fracturing $k^{\prime}$.'was taken. The velocity of the wave propagation in the methane-air medium is considered as $V_{\phi}$; as $V_{\Pi}$ - the wave propagation velocity in the rock mass before hydraulic fracturing $V_{1}$; as $V_{p}$ - the velocity of wave propagation in the massif after hydraulic fracturing. As a result, the expression (2) has taken the form [9]: 


$$
\frac{1}{V_{2}}=\frac{k^{\prime}}{V_{\phi}}+\frac{1-k^{\prime}}{V_{1}}
$$

At the same time, the choice of the massif primary fracturing, not changed by the hydraulic fracturing, was assumed based on geological exploration data. In the case of their absence, fracturing was taken as the ratio of cracks and voids volume in the studied massif to the bulk of rock skeleton from expression (2) with the replacement of $V_{p}$ by $V_{1}$ [ [9]:

$$
\frac{1}{V_{1}}=\frac{k}{V_{\phi}}+\frac{1-k}{V_{\Pi}} .
$$

From expressions (3) and (4) the known values of the velocities were determined by $k^{\prime}$ 'and $k$, respectively. Thus, the fracture characteristic was calculated after hydraulic fracturing $\mathrm{k} k^{*}[9]$ :

$$
k^{*}=k \cdot k^{\prime}
$$

In practice, when assessing the parameters of hydraulic impact at operating mines, the use of the selected criterion, in the absence of the possibility of comparison with geological and actual data, led to the formation of coefficients that quite abstractly described the degree of change in the geomechanical characteristics of the coal-rock mass. To solve this problem, a method for assessing the degree of fracturing of the massif was adapted, based on the geophysical index characterizing it [10]:

$$
B_{e}=\left(V_{P 0} / V_{P i}\right)^{2}-1
$$

where $V_{P i}$ is the velocity of the longitudinal wave propagating along the trajectory $\mathrm{i}$; $V_{P 0}$ - the speed of the longitudinal wave in the individual elements that make up the array.

An important feature of this method is a clear gradation of the degree of fracturing according to 6 categories used in the classification of the Interdepartmental Commission on Blasting: 1 - extremely fractured, 2- highly fractured, 3-medium fractured, 4-weakly fractured, 5-safe, 6-tense [10]. Taking into account that the tomographic survey was carried out for a section of a massif, divided in accordance with the parameters of the applied measurement scheme into $\mathrm{N}$ pixels, then to find the geophysical index in each pixel, into which, among other things, individual fractured elements of the massif are divided, expression (6) took the form:

$$
B_{e j}=\left(V_{P 0 \mathrm{j}} / V_{P i j}\right)^{2}-1
$$

To assess the effectiveness of the considered method to assess fracturing, geophysical studies were carried out in the active longwall in one of Kuznetsk coal basin mines. Within the framework of scientific and technical work, a survey of a 300x220 m coal-rock massif was carried out before and after hydraulic fracturing.

\section{Results and Discussion}

Based on seismic measurements results, horizontal geophysical sections of the velocity characteristics distribution in the active roof area were obtained before and after hydraulic fracturing (Figure 2). On the formed sections propagation velocities of longitudinal seismic waves were estimated. 
(a)

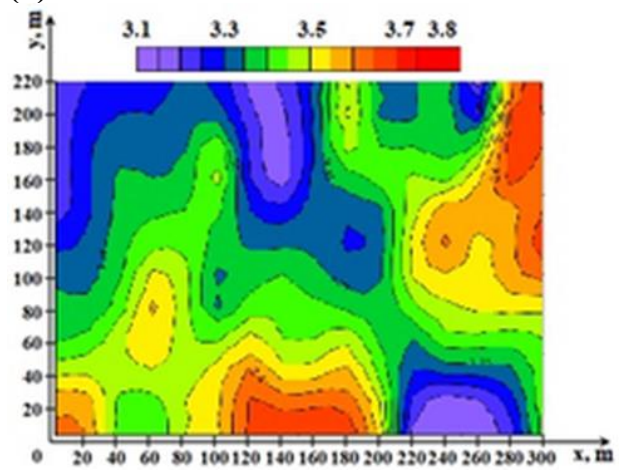

(b)

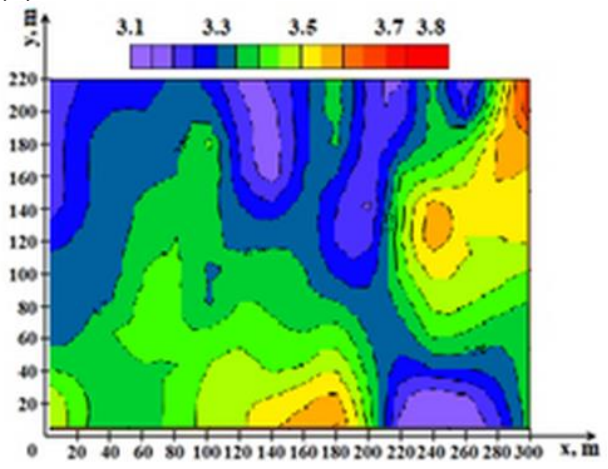

Fig. 2. Horizontal seismic tomographic sections by longitudinal velocities (a) before hydraulic fracturing, (b) after hydraulic fracturing.

It was determined that before the hydraulic impact, the active roof was characterized by the velocities of longitudinal waves propagation of 3.1-3.8 km / s (Figure 2 a), which indicated a predominantly medium-stable state of its constituent rocks. Within the measurement area, areas of minimum velocities $(<3.2 \mathrm{~km} / \mathrm{s})$ were identified, which are probably areas of increased fracturing relative to the rest of the investigated area of the extraction column. Taking into account that velocity values were not extremely low, the probability of an unstable roof in these zones was assessed as low. Also, in the active roof, areas of maximum velocities $(>3.60 \mathrm{~km} / \mathrm{s}$ ) were recorded, containing more durable rocks in a stressed state, potentially capable of being local areas of the main difficult to break roof. According to seismic measurements results undertaken after hydraulic impact (Figure $2 \mathrm{~b}$ ), a change in the active roof state was recorded, expressed in a general decrease in the velocity background in the study area, as well as in area increase in minimum velocities sections and in the area reduction of maximum velocities sections. The registered changes in the characteristics of the active roof indicate its softening. The registered changes in the characteristics of the active roof indicate its softening. To assess the unloading degree of the active roof based on software calculation procedures application, a seismotomographic cross section of the calculated value was formed, determined by the ratio of velocities variations recorded in the measurements before and after hydraulic fracturing to the range of their possible change.

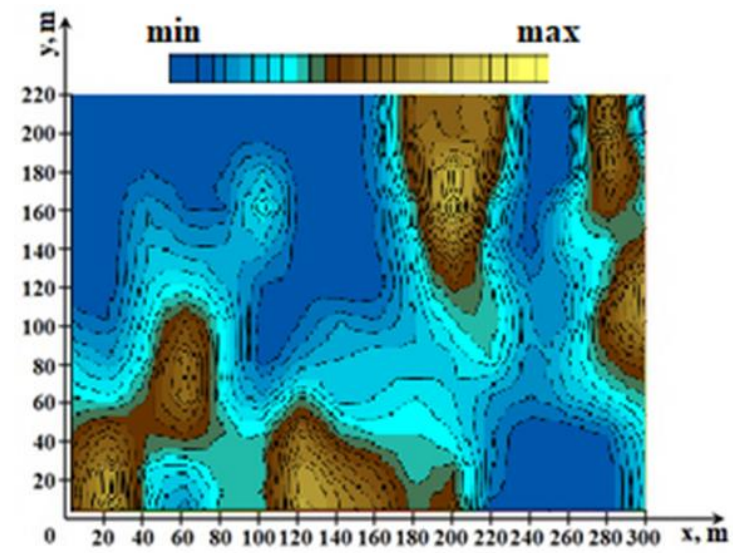

Fig. 3. Horizontal seismic tomographic section, reflecting the degree of active roof unloading, recorded in the measurements before and after hydraulic impact. 
The obtained horizontal section was characterized by presence of sections in the active roof, with a change in the calculated ratio within $10-25 \%$. Besides, almost everywhere within the measurement area, a change in the ratio of up to $10 \%$ was recorded. This testifies the fact of happened unloading, caused by a combination of natural and man-made reasons: the initial strength and structural characteristics of the roof rocks, the influence of mining, drilling of boreholes well, as well as the consequence of hydraulic impact on the contained coal seam. Also, the change in the ratio under consideration was to some extent caused by the natural unloading of the massif, which occurred in the interval between the first and second measurements.

Due to the lack of geological information on the investigated area fracturing, the application of the approach based on the relations (2-5) would not provide the final set of data in a format accessible to their end users (geological engineers and officers of coal mining enterprises). To bring the results to a form corresponding to the classification, of the Interdepartmental Commission on Blasting. Based on dependence (7), the values of the geophysical index $B_{e}$, characterizing the massif fracturing were determined [10]. Based on the results of $B_{e j}$ comparison with the classifier, horizontal seismotomographic sections were formed, reflecting the active roof rocks distribution by fracture classes before and after hydraulic impact on the roof (Figure 4).

(a)

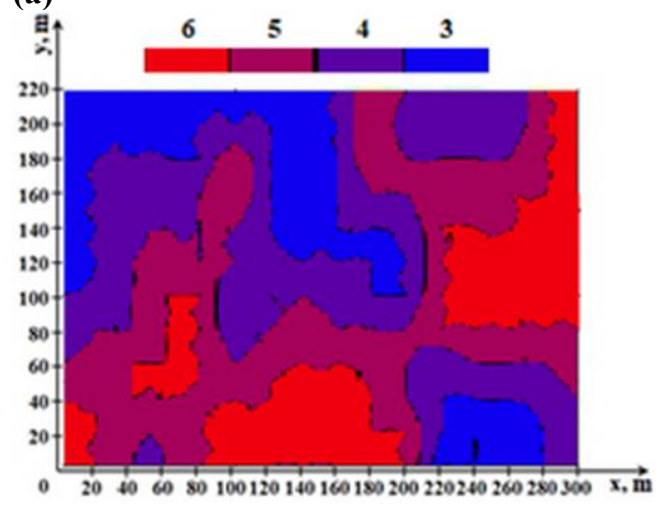

(b)

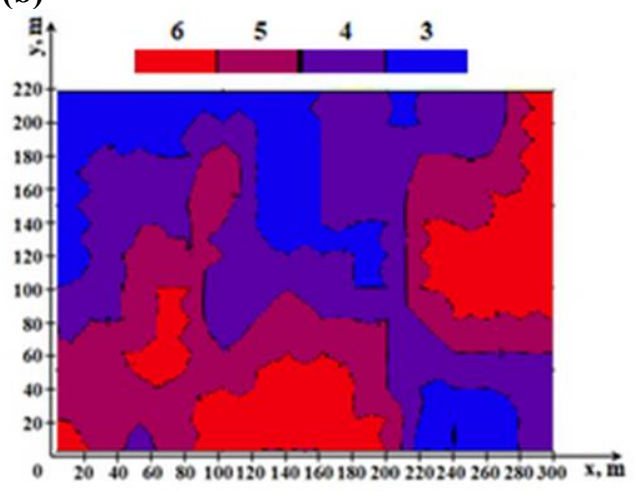

Fig. 4. Horizontal seismic tomographic sections reflecting the distribution of active roof rocks by fracture classes: (a) before hydraulic fracturing, (b) after hydraulic fracturing.

During the estimate, it was determined that within the measurement site, the active roof was predominantly in a safe or stressed state before the hydraulic fracturing (Figure 4a). The location of areas with rocks in a stressed state generally corresponded to position of areas of maximum velocities that contained strong rocks in a stressed state (Figure 2a). 


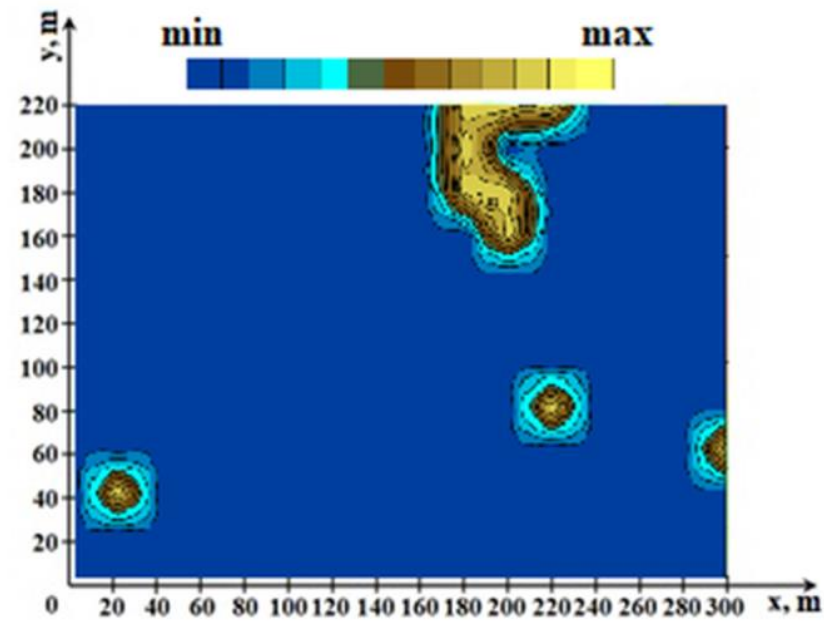

Fig. 5. Horizontal seismic tomographic section, reflecting the degree of change in the rocks fracturing class of the active roof based on hydraulic impact results.

It was also found that areas of increased fracturing relative to the rest of investigated areas of the extraction column (Figure 2a) can be classified as zones with medium fracturing of rocks (Figure 4a). The fixed design parameters of the active roof fracturing, distributed in the interval 3-6, have good convergence with the generalized geological data on the mine allotment. On the horizontal seismic tomographic section after hydraulic fracturing (Figure 4b), significant changes relative to the identified classes were not noted. Visually, the change in classes is manifested mainly in the formation of weak fracturing within areas of intact rocks. To assess the recorded fracturing by the indicators difference before and after hydraulic impact, a horizontal seismotomographic section was plotted, reflecting the degree of change in the fracture class in the measurement area (Figure 5). The indicated section demonstrates that almost everywhere the massif unloading under the hydraulic impact hasn't induced a change in rocks fracturing class of the active roof. Nevertheless, within the measurement area, 4 sites of the analyzed value change are recorded by 1 point.

\section{Conclusion}

In general, the results of undertaken measurements indicate the expediency of the applied approach to estimate hydraulic fracturing efficiency determination. According to the results of performed studies it can be noted that the calculated fracturing values of the coal-rock massif obtained on the basis of mine geophysical information are in good convergence with generalized geological data of the mine allotment. This confirms the fundamental possibility of using the approach to assessing the fracturing degree [10] in operating coal mines. At the same time, the approach, obviously, requires improvement in terms of comparing the classification of the rock massif fracturing degree with actual values of active roof fracturing on the excavation sites. In addition, the approach requires supplementation in relation to the state assessment of the coal seam itself. With proper indicators correction based on actual data, a transition to the fracturing characteristic determined by the ratio (5) is possible. As can be seen from Figures 3 and 5, with the already confirmed unloading degree of the massif, by general categories of classification of the Interdepartmental Commission on Blasting, observed changes are insignificant. In this 
case, the $\mathrm{k} *$ characteristic can be used as a parameter characterizing the relative change in rock fracturing within individual classes.

\section{References}

1. B. Huang, Q. Cheng, X. Zhao, C. Kang. Journal of Geophysics and Engineering, 15, 6, 2492 (2018)

2. T. Katsaga, A. Riahi, D.O. DeGagne, B. Valley \& B. Damjanac. Hydraulic fracturing operations in mining: conceptual approach and DFN modeling example 255 (2015)

3. E.J. Nelson, S.T. Chipperfield, R.R. Hillis, J. Gilbert, J. McGowen, and S.D. Mildren / The relationship between closure pressures from fluid injection tests and the minimum principal stress in strong rocks, 44, 787 (2007)

4. L. Cheng, Z. Ge, J. Chen, L. Zou, Y. Cheng, S. Xiao. Hydraulic fracturing and its effect on gas extraction and coal and gas outburst prevention in a protective layer: a case study in China, 23, 4, 427 (2020)

5. K. Luxbacher, E. Westman, P. Swanson, M. Karfakis, Three-dimensional time-lapse velocity tomography of an underground longwall panel, 45, 4, 478 (2008)

6. Z. Wang, X. Li, D. Zhao, X. Shang, L. Dong Time-lapse seismic tomography of an underground mining zone, 107, 136 (2018)

7. V.I. Klishin, O.V. Taylakov, G.Yu. Opruk, M.P. Makeev, S.V. Sokolov, A.S. Teleguz, and A.L. Tatsienko. Seismic monitoring of hydrodynamic impact on coal seam at interval hydraulic fracturing, 377, 1 (2019)

8. M.J. Jackson, and D.R. Tweeton MIGRATOM-Geophysical tomography using wavefront migration and fuzzy constraints Report of Investigations RI 9497 Department of the Interior, US Bureau of Mines, Washington, DC (1994)

9. V.I. Klishin, O.V. Taylakov, G.Yu. Opruk, S.V. Sokolov, A.V. Nikolaev Assessment of elastic seismoacoustic vibration propagation through coal and rock mass within the extraction column during directional hydraulic fracturing (DHF) implementation, 206, 1 (2018)

10. N.N. Abramov. Estimation Procedure of Influence Exerted by Trigger Effects in Rock Mass on Technical Condition of Long-Term Operated Underground Structures, 54, 5, 709 (2018) 